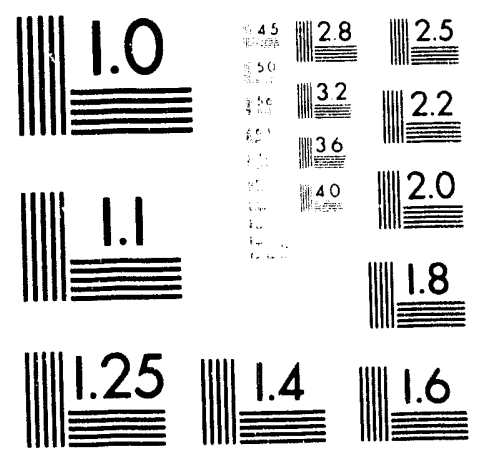



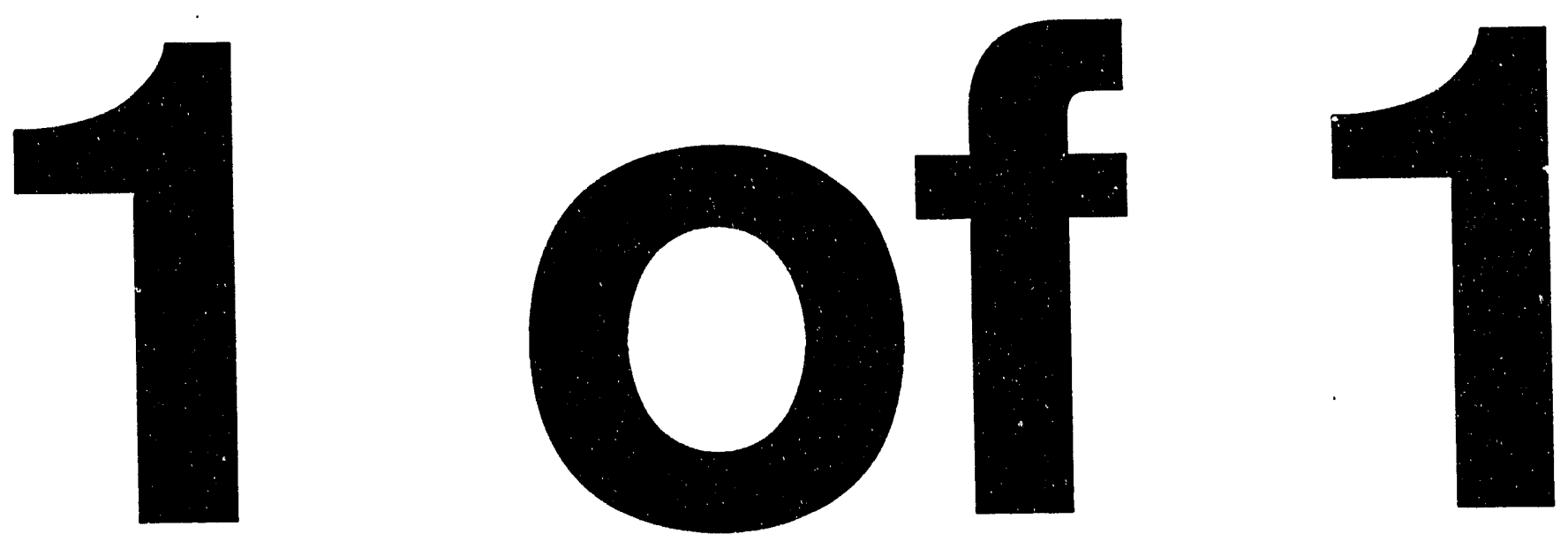


\title{
A Statistical Study of the Performance of a Heat- Flow Calorimeter: Calibration and Error Analysis
}

\author{
Michael C. Axelrod \\ Lawrence Livermore National Laboratory \\ Electronics Engineering
}

October 1991

MASTER 


\begin{abstract}
From an analysis of more than three years of calibration data we derived a new calibration data for the "small" calorimeter. Using this calibration we should be able to measure the thermal output power of unknown radiological sample to better than $0.3 \%$ of the true value (based on one standard deviation) when the unknown lies in the power range $0.25-4.0$ watt. We learned that the calibration data taken before August 1987 showed considerably more variability than data collected subsequently. We also detected a long-term periodic fluctuation in the data the scale of about one year. The cause of this fluctuation is unknown. In fluctuation does significantly reduce the accuracy of our measurements. The aceuracy is of thecalorimeter is degraded by the random error of the entire measurement system, the periodic fluctuation, and the statistical error in estimating the calibration coefficients. We recommend that the cause of the periodic fluctuation be determined by monitoring the environmental conditions of the calorimeter laboratory, and a continuous program of recalibration to assure continued accuracy of these measurements.
\end{abstract}

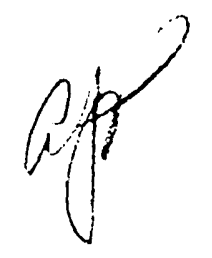




\section{Administrative Information}

The work was supported by the Safeguard and Security Program, Project Leader: David Parks. The author is located in the Signal and Image Processing Research Group, Engineering Research Division, Electronics Engineering 


\section{SUMMARY AND CONCLUSIONS}

From an analysis of more than three years of data for the "small" calorimeter, we derived a new calibration function for this instrument. The thermal power $w$ (in watts) of an unknown sample is calculated from the equilibrium output voltage $v$ by the following formula:

$$
\hat{w}=\frac{1}{2 \hat{\beta}_{2}} \sqrt{\hat{\beta}_{1}^{2}+4 \hat{\beta}_{2}\left(v-\beta_{0}\right)}-\frac{\hat{\beta}_{1}}{\hat{\beta}_{2}}
$$

where

$$
\begin{aligned}
& \beta_{0}=0.012385287 \\
& \beta_{1}=5.474903380 \\
& \beta_{2}=-0.008164486
\end{aligned}
$$

The calculation of the one-sigma measurement error, expressed as a fraction of the measured power, depends on a complex mathematical formula derived in this report. From this formula, we can assert that small-calorimeter power measurements should have an error of no more than $1 / 3$ percent when the power output of the sample is greater than $1 / 4$ watt $(W)$ and less than $4 \mathrm{~W}$ (see Table 1).

Table 1 Calculated measurement error by thermal power.

\begin{tabular}{|cc|}
\hline $\begin{array}{c}\text { Thermal Power } \\
\text { W }\end{array}$ & $\begin{array}{c}1 \sigma \text { Random Error } \\
\text { (percent) }\end{array}$ \\
\hline 0.25 & 0.10 \\
0.50 & 0.09 \\
1.00 & 0.10 \\
2.00 & 0.17 \\
4.00 & 0.31 \\
10.00 & 0.80 \\
20.00 & 1.65 \\
\hline
\end{tabular}

Above $1.5 \mathrm{~W}$, the error increases linearly with power. However, since we have no calibration data above $4 \mathrm{~W}$, the errors quoted in Table 1 for ten and twenty watts might be incorrect.

The following comments summarize what we learned from the analysis.

- The data before August 1987 exhibited considerably more scatter than the data collected after that date. We eliminated this early data from the study.

- We detected a (nearly) sinusoidal fluctuation in the voltage measurements on the scale of a year. Using a periodogram analysis, we found that the amplitude of the fluctuation rapidly increases with the power. For example, the fluctuation has an amplitude of $6 \mu \mathrm{V}$ at one watt growing to $36 \mu \mathrm{V}$ at four watts. On the other hand, the period of the fluctuation 
changes little with increasing power, 343 days at one watt compared to 330 days at four watts. As yet, we do not know the cause of this fluctuation.

- With high accuracy, we can predict the equilibrium output voltage of the calorimeter by using a quadratic polynomial function of power. The calibration function is the inverse of this polynomial.

- The error in measuring power depends on the following:

1. The amplitude of the sinusoidal fluctuation

2. The random error of the measurement system

3. The error in estimating the coefficients of the calibration function

We used three statistical models to quantify the contributions of each of the above to the total measurement error.

- If our statistical model of the calorimeter is correct, then the new calibration function should provide statistically unbiased estimates the thermal power outputs of unknown samples.

- Our estimates of measurement error strongly depend on the accuracy of the radiometric standards provided by Mound Laboratories. If Mound has not provided accurate correction tables for the standards then our results are not valid.

- Our estimates of accuracy for fu'cure measurements depend on the stability of laboratory conditions. If the small calorimeter is modified, or if experimental procedures should change, then the accuracy of the measurements will change from what is stated here.

- We do not know the cause of the periodic fluctuation. We do not know if the amplitude of the fluctuation will change at some time in the future. As far as knowing the accuracy of our power measurements, we are at the mercy of whatever produces this fluctuation. This is not a comfortable condition to be in.

- The measurement accuracy would improve dramatically at the higher powers if we could eliminate the periodic fluctuation. For example, at four watts the fluctuation increases the error by a factor five. 


\section{Recommendations}

- We should attempt to determine the cause of the long-term periodic fluctuations detected in the voltage data. If we could eliminate this effect, we would significantly improve the accuracy of our small-calorimeter measurements.

- We should make measurements on standards with power levels botow $\theta .25$ and above $4.0 \mathrm{~W}$ to see how well the calibration equation works outside the range of values considered in this report.

- The calibration coefficients should be updated as new measurements on standards are made.

- We should record the following additional information when a radiometric standard is run:

1. The temperature and humidity during the time interval the voltage takes to reach its equilibrium value.

2. The name(s) of the operator (s) performing the calibration run

3. A reference to the Mound Laboratories correction tables used to calculate the power level of the standard under test.

- If possible, we should verify the radioactive decay corrections supplied by Mound Laboratories. 


\section{Introduction}

This report presents the results of a detailed statistical analysis of calibration data from a twin-chamber, steady-state, heat-flow calorimeter that was designed and built at Lawrence Livermore National Laboratory (LLNL). We usually refer to this instrument as the "small" calorimeter, or "calorimeter 32 ." We calibrate the instrumentby messurements on a set of radiometric standards supplied by Mound Laboratories that have thermal power outputs at $1 / 4,1 / 2,1,2$, and $4 \mathrm{~W}$ (all values are nominal). We have data taken over a period of nearly three years, almost 200 separate runs. This data is a valuable resource because a single run often takes more than one day. This study attempts to make the best use of all the available data to achieve a calibration that will provide power measurements of high accuracy.

First, we will formulate the calibration problem mathematically and propose a statistical model to explain the data. We then refine and enlarge the model according to the results of a series of statistical diagnostic procedures. Finally, we will derive a new calibration and quantify its performance in terms of an error analysis.

This report considers only the statistical issues about the calibration of the calorimeter, we refer the reader to Steward and Tsugawa (1975) and to Gunn (1966) for a physical description of the calorimeter and details on its theory and method of operation.

\section{Mathematical Formulation of the Calibration Problem}

The "small" calorimeter measures the thermal power output of a radiometric sample when thermal equilibrium is reached between the sample under measurement and a reference heat-source. We conclude that thermal equilibrium occurs when the voltage from some thermopile sensor stops changing, or reaches a minimum rate of change (usually within 24 hours). We refer to the voltage at thermal equilibrium as the equilibrium voltage. When we speak of variations or fluctuations in the equilibrium voltage we mean changes that occur over different measurement runs and not changes that occur during a run. The equilibrium voltage at run number $i$ is $v_{i}$. The time associated with run $i$ is $t_{i}$, measured in days from an arbitrary origin to the time the sample is removed from the calorimeter. Each of the radiometric standards experiences radioactive decay; thus multiple measurements on a given standard are really measurements of different thermal power levels. Mound Laboratory provides tables that give the thermal power output of a standard on differnt days. We assume that these tables contain values accurate to the stated precision.

Following the recommendation in ANSI N15.22-1987, we express the equilibrium response voltage $v_{i}$ to the thermal power $w_{i}$ by the following regression model:

$$
v_{i}=\beta_{0}+\beta_{1} w_{i}+\beta_{2} w_{i}^{2}+\varepsilon_{i} \quad i=1,2, \ldots N
$$

where $\beta_{0}, \beta_{1}$ and $\beta_{2}$ are unknown coefficients, and the $\varepsilon_{i}$ are random variables representing random variations in the response voltage $v_{i}$. The quantity $N$ is the total number of measurements available. In Eq. (1) the $w_{i}$ are not random in the sense that repeat measurements of thermal power on the standard with a perfect instrument (no error) would give identical values, and we know that value. In practice, this means that the uncertainty of 
the $w_{i}$ is very small. On the other hand, the $v_{i}$ are random. Evidently the random nature of the $v_{i}$ is due to some exogenous disturbance or random variation in the properties of the hardware components of the calorimeter, not the error of measurement, because the digital voltmeter is apparently very accurate.

We invert Eq. (1) to predict the power from voltage ${ }^{1}$ :

$$
\hat{w}=\frac{1}{2 \hat{\beta}_{2}} \sqrt{\hat{\beta}_{1}^{2}+4 \hat{\beta}_{2}\left(\nu-\beta_{0}\right)}-\frac{\beta_{1}}{2 \beta_{2}}
$$

The $\beta_{2}$ coefficient appearing in Eqs. (1) and (2) represents a small but significant correction to a linear calibration. We can see the correction explicitly if we expanc the right hand side of Eq. (2) in an Taylor Series and recain only the terms up to second order:

$\hat{w} \approx \frac{v-\beta_{0}}{\beta_{1}}-\frac{\beta_{2}}{\beta_{1}} \frac{\left(v-\beta_{0}\right)^{2}}{\beta_{1}^{2}}$

If we set $\beta_{2}=0$ in Eq. (3) then we see that power is a linear function of voltage, therefore, $\beta_{2}$ determines the amount of second order correction to a linear calibration. From Eq. (3), the correction at one watt is essentially the ratio $\beta_{2} / \beta_{1}$. This ratio is $0.15 \%$, a value close to the value in Table 1 for the statistical error at one watt. Therefore, to achieve errors commensurate (or better) with statistical errors quoted in Table 1, the second order correction is essential. What we have shown here is that we cannot achieve high accuracy with a linear calibration.

\section{Analysis of Residuals}

If we assume that the random errors $\varepsilon_{i}$ from Eq. (1) are statistically independent and identically distributed with zero mean, then we have the standard linear regression model where the coefficients $\beta_{0}, \beta_{1}$ and $\beta_{2}$ are estimated by an ordinary (unweighted) least squares procedure. However, we need to analyze the residuals to verify that those assumptions hold. From Eq. (1) the regression residuals are:

$\hat{\varepsilon}_{i}=v_{i}-\hat{v}_{i}$

where $\hat{v}_{i}=\hat{\beta}_{0}+\hat{\beta}_{1} w_{i}+\hat{\beta}_{2} w_{i}^{2}$.

\footnotetext{
${ }^{1}$ A comment on notation. The circumflex " $\wedge$ " placed over a parameter denotes the estimate of the parameter in a statistical model. The parameter is an unknown but fixed quantity and therefore not random. On the other hand, the estimate of a parameter is random because it is a function of random data. For typographical convenience, we will not always use the circumflex when the meaning is obvious from context.
} 
A plot of the regression residuals (for a power level of about $1 \mathrm{~W}$ ) against time reveals considerable scatter before late 1988 indicating that the measurement process was not in control. We eliminate these points as not representing of current laboratory conditions. The remaining points when plotted against time show a cyclical pattern with a period of about one year (see Fig. 1). The solid curve in Fig. 1 is a smoothed version of the points using a robust smoother first proposed by Cleveland (1979). The same pattern appears in the residuals at the other levels of thermal power, but at a greater amplitude.

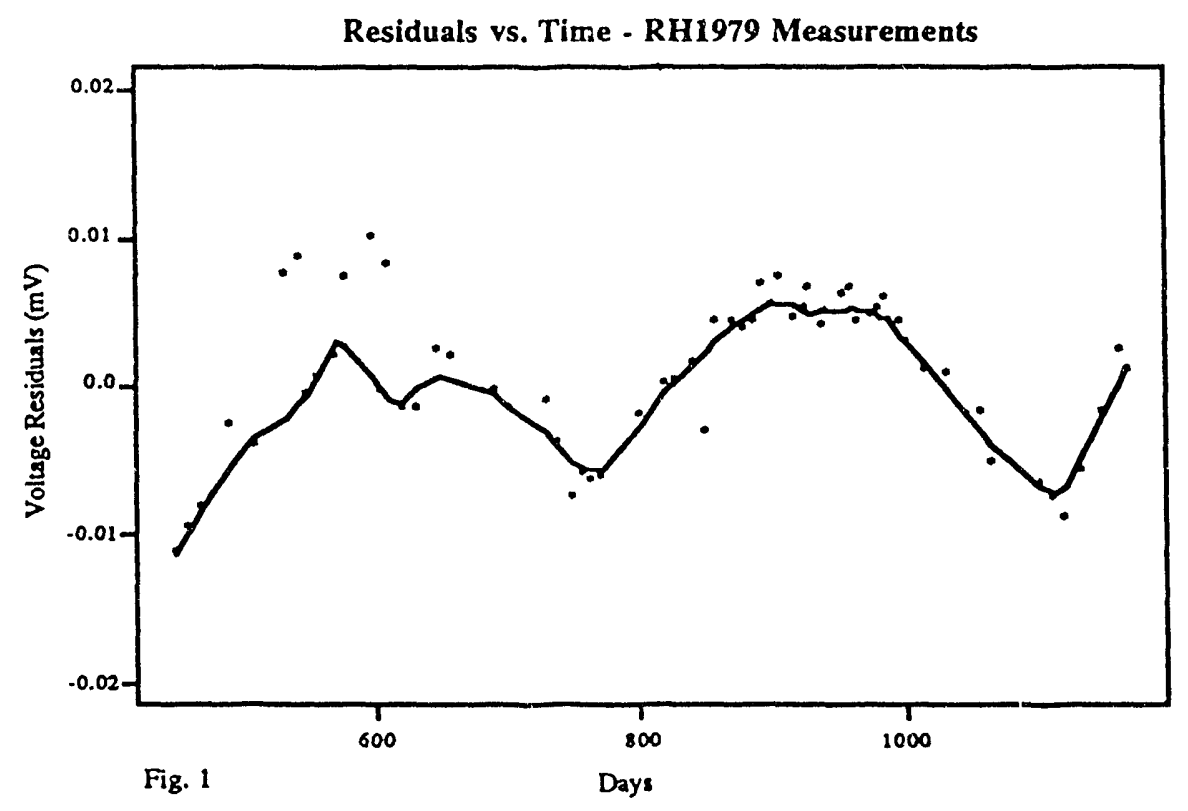

We can enlarge the model given by Eq. (1) to include a sinusoidal term with amplitude $A$, period $T$, and phase angle $\phi$ (see Eq. (4)).

$v_{i}=\beta_{0}+\beta_{1} w_{i}+\beta_{2} w_{i}^{2}+A \sin (2 \pi t / T+\phi)+\varepsilon_{i}$

The estimation of the coefficients in Eq. (4) is complicated because the equation is nonlinear in $T$ and $\phi$. However, if we expand the sin term, we get Eq. (5) which is only nonlinear in $T$. This equation is easier to deal with.

$v_{i}=\beta_{0}+\beta_{1} w_{i}+\beta_{2} w_{i}^{2}+\beta_{3} \sin (2 \pi t / T)+\beta_{4} \cos (2 \pi t / T)+\varepsilon_{i}$

A simple way to determine the period $T$ is to adapt the least squares periodogram technique suggested by Bloontield (1976) and make multiple fits of Eq. (5) to the data with different trial values of $T$. We then select the value of $T$ that maximizes the absolute value of the amplitude $A$, given by:

$$
|A|=\sqrt{\beta_{3}^{2}+\beta_{4}^{2}}
$$


The periodogram for the data from the one-watt (RH1979) standards data is given in Fig. 2. From Fig. 2, the peak amplitude is about $6 \mu \mathrm{V}$ and the period is 343 days. If we apply this technique to the data from the four-watt standard, we find the peak amplitude is about $36 \mu \mathrm{V}$ and the period is 330 days. These results strongly suggest that some periodic or quasi-periodic fluctuation exists in the measurement system where both the period and amplitude depend on the thermal power level of the sample under measurement.

Unfortunately, we do not have sufficient data to compute periodograms for the other power levels. From now on, we assume that the amplitude of the fluctuation grows as the square of the power.

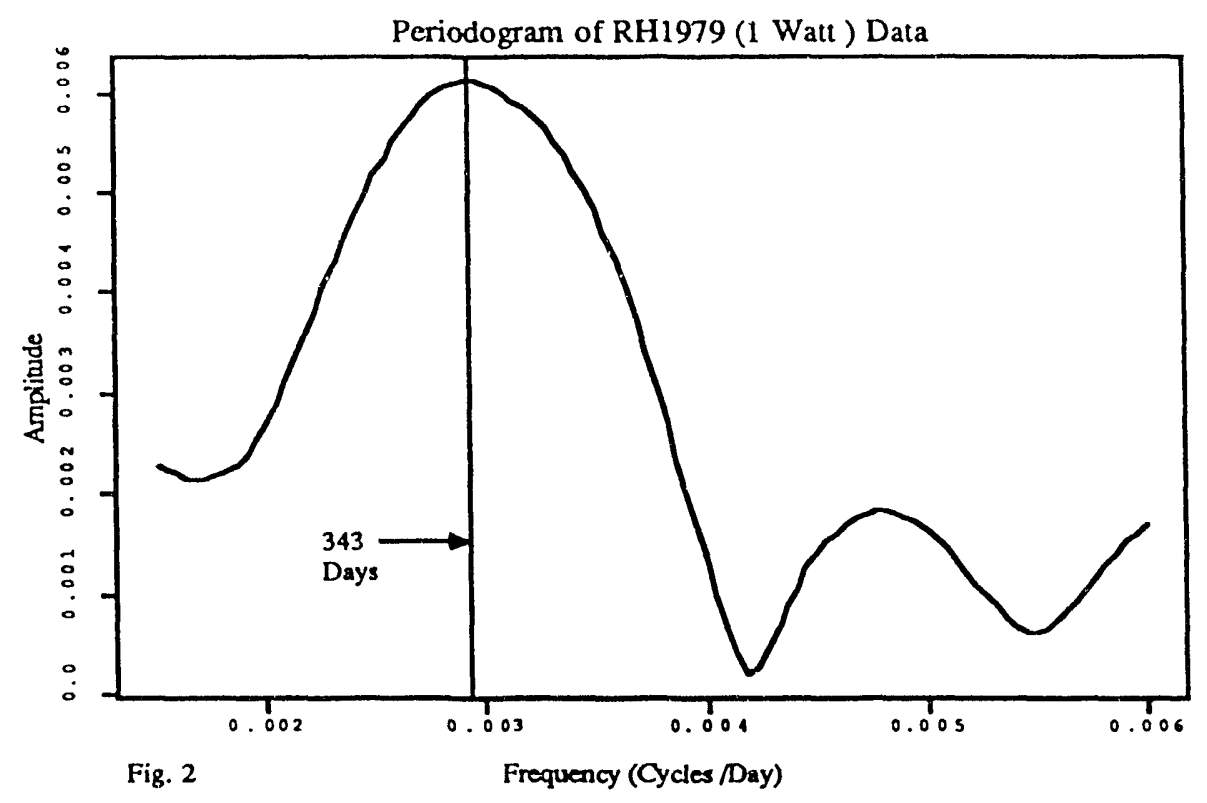

A regression model that incorporates this assumption is Eq. (6); the parameter $a$ is a measure of the strength of the cyclic fluctuation. Strictly speaking, this model is valid for only a single power level because the period changes with power as well as the amplitude. However, for the calibration data we assume one frequency is dominant with a corresponding period of 343 days.

$v_{i}=\beta_{0}+\beta_{1} w_{i}+\beta_{2}(1+a \sin (2 \pi f t+\phi)) w_{i}^{2}+\varepsilon_{i}$

A more accurate model would contain multiple periodicities, but the one frequency model should remove most of the deterministic structure of the residuals. From the periodogram analysis, we know that the value of the dimensionless parameter $a$ in Eq. (6) is approximately 0.4 .

Next, we examine the homogeneity of the error variances after recomputing the residuals using the regression model given by Eq. (6). A plot of the voltage residuals versus the fitted values of voltage (see Fig. 3 ) shows the variance of the residuals increases with the level of thermal power. This effect would be in addition to the increase in amplitude of the cyclic fluctuation since we have already removed that effect with the new 
model. The vertical groupings on the plot correspond (from left to right) to the nominal power levels of the standards: 1/4,1/2,1, RH1979, 2, and 4 watts. The residuals were computed assuming the error variance was homogeneous, and the effect of violating this assumption is to reduce the efficiency of the estimates of the parameters of the model. ${ }^{2}$

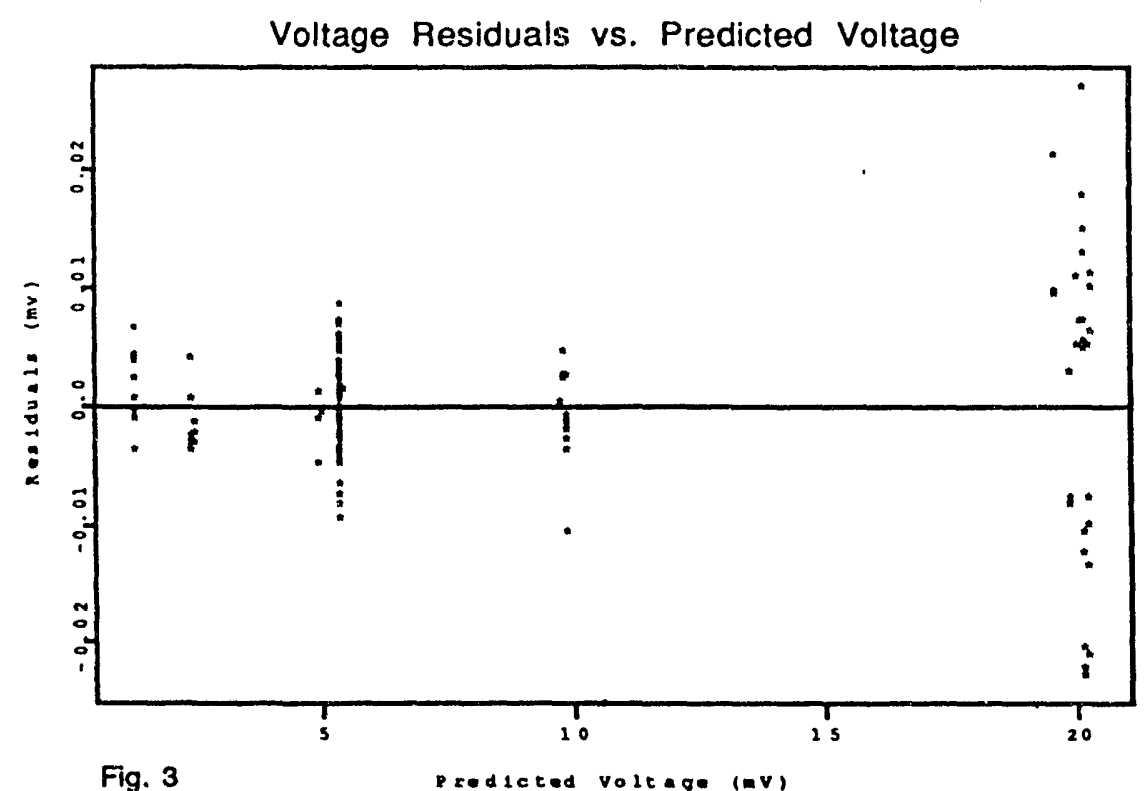

We need to find some reasonable functional relationship between the variance of the voltage residuals and the thermal power level. Since voltage and thermal power are nearly linearly related, we can group the voltage residuals by the nominal power level of the group, and examine the spread within each group. A useful graphic for this purpose is the boxplot suggested by Tukey ${ }^{3}$ (1977). Boxplots provide a concise summarization of the distributional properties of the data within a group by means of a special icon. A boxplot of the residuals is shown in Fig. 4. The horizontal line-segment within the rectangular portion of the icon represents the median value of the data; the top and bottom of the rectangle represent the upper and lower quartiles of the data; the extreme values are plotted at the ends of the vertical dashed lines extending from the top and bottom of the rectangle. Finally, possible outliers are plotted as isolated points. Thus, the height of the rectangle gives us an idea of the spread of the data in each group. We can see significant

\footnotetext{
${ }^{2}$ The estimates will still be unbiased if the form of the model is correct.

${ }^{3}$ See Graphical Methods for Data Analysis, (Chambers et al 1976) for a description of the boxplots used in this report.
} 
distributional asymmetry in most of the groups because the median is not centered. There is a possible outlier in the half-watt group.

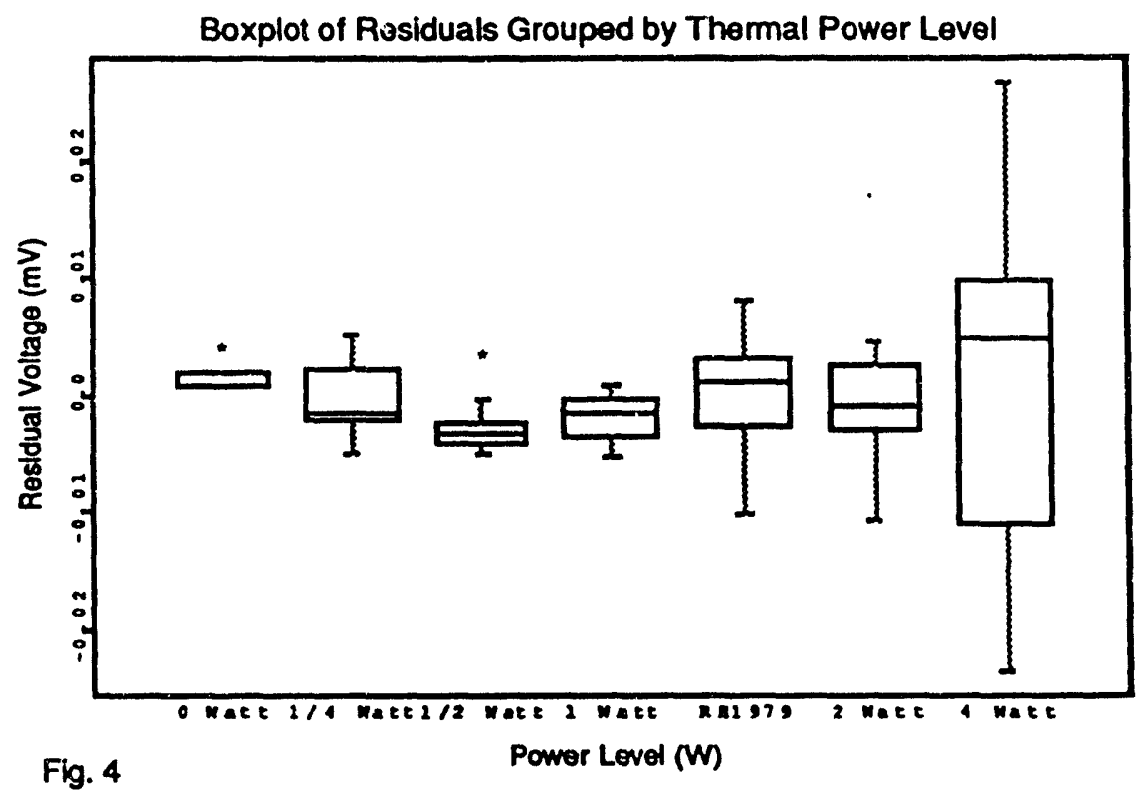

Since the distributions are not normal, we should use robust measures of spread instead of the standard deviation. One robust replacement for the standard deviation is the $F$-pseudosigma statistic (see Hoaglin 1983), calculated from the interquartile range by dividing by 1.349 . When the data are normal, the $F$-pseudosigma will approximately equal the standard deviation. The F-pseudosigma appears to increase linearly with the power level (see Fig. 5) suggesting we fit a line of the form:

$s_{i}=\alpha_{0}+\alpha_{1} w_{i}$

The F-pseudosigma is itself a random quantity with a variance, and this variance is not constant (see Table 2). As a result, we need use weighted least squares in fitting the line, where the weights are proportional to the reciprocal of the variances. The theoretical variance of the F-pseudosigma statistic is not available in closed form, so we will use the variance of the sample standard deviation $s$ for normal data (see Kendall and Stuart) as an approximation:

$\operatorname{Var}(s)=\frac{\sigma^{2}}{2 n}$ 
Table 2

Srread and Related Statistics for Residuals by Nominal Power Level

\begin{tabular}{|lcccc|}
\hline \multicolumn{1}{|c|}{$\begin{array}{c}\text { Nominal } \\
\text { Thermal Power }\end{array}$} & $\begin{array}{c}\text { Number } \\
\text { of Points }\end{array}$ & $\begin{array}{c}\text { Pseudo } \\
\text { Std. Dev. (mV) }\end{array}$ & $\begin{array}{c}\text { Variance of Pseudo } \\
\text { Standard Dev. }(\mu \text { 2 } 2)\end{array}$ & $\begin{array}{c}\text { Relative } \\
\text { Weight }\end{array}$ \\
\hline O Watt & 8 & 0.7413 & 0.0343 & 0.563 \\
1/4 Watt & 13 & 3.2128 & 0.3970 & 0.049 \\
1/2 Watt & 9 & 1.2930 & 0.0929 & 0.208 \\
1 Watt & 4 & 2.3726 & 0.7036 & 0.027 \\
RH1979 (1 Watt) & 63 & 4.4533 & 0.1574 & 0.123 \\
2 Watt & 11 & 4.1338 & 0.7767 & 0.025 \\
4 Watt & 31 & 15.3392 & 3.7950 & 0.005 \\
\hline
\end{tabular}

The points near zero power get the most weight, while the four-watt point gets the least (see Table 2). Nevertheless, the four-watt point does significantly affect the slope of the titted line (see Fig. 5) because it is an end-point ${ }^{4}$. Here is an example where it might be unwise to fit the regression line by eye because the result of these competing effects is not obvious.

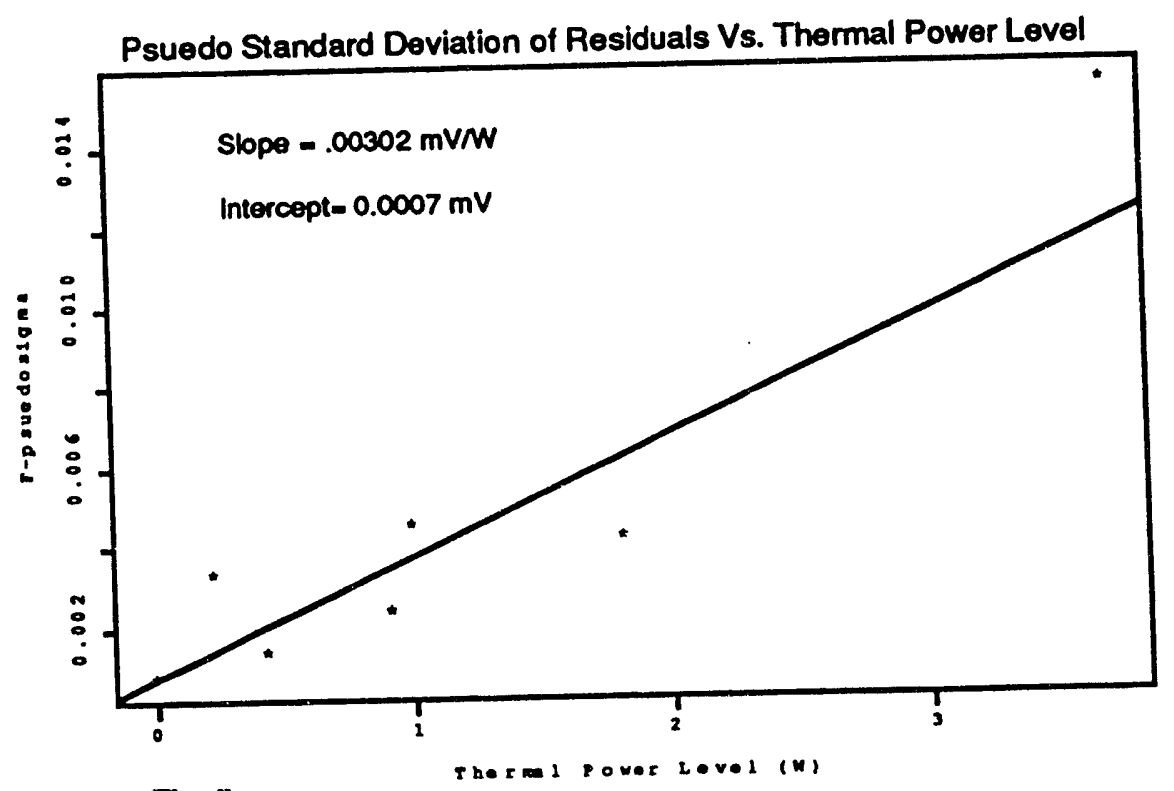

Fig. 5

We now have a relationship between the variance of the random errors in Eq. (6) and power:

$$
\sigma_{\varepsilon_{i}}^{2}=\left(\alpha_{0}+\alpha_{1} w_{i}\right)^{2}
$$

${ }^{4}$ Such a point is known as a high leverage point in the statistical literature. See Cook and Weisberg (1982). 


\section{Fitting the Model}

We refit the model using weighted regression and a period of 343 days. The weights are the reciprocals of the random error variances.

$v_{i}=\beta_{0}+\beta_{1} w_{i}+\beta_{2} w_{i}^{2}+\beta_{3} w_{i}^{2} \sin (2 \pi t / T)+\beta_{4} w_{i}^{2} \cos (2 \pi t / T)+\varepsilon_{i}$

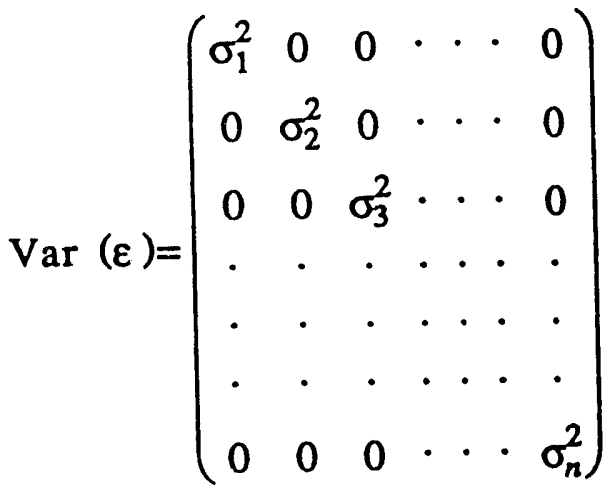

where $\varepsilon$ is a vector of residuals:

$$
\varepsilon=\left[\begin{array}{c}
\varepsilon_{1} \\
\varepsilon_{2} \\
\cdot \\
\cdot \\
\varepsilon_{n}
\end{array}\right]
$$

Although other laboratories have also detected a periodic fluctuation in their calorimeter measurements, apparently no one has identified the cav $\mathrm{e}^{5}$. Since we cannot assume that the signal will persist, it would not be prudent to incorporate the sinusoidal terms in Eq. (7) into the final calibration equation. However, we do need to use Eq. (7) to get unbiased estimates of $\beta_{0}, \beta_{1}$ and $\beta_{2}$. In theory, the estimates using Eq. (7) should give the same values as if the periodic signal were to vanish.

${ }^{5}$ Private communication from David Parks. 
Table 3

Estimates of Regression Coefficients from Eq, (7) and Associated Statistics

\begin{tabular}{|cccc|}
\hline Coefficient & Value & Variance $\times 10^{-6}$ & \multicolumn{1}{c|}{ statistic } \\
\hline$\beta_{0}$ & 0.012385287 & 0.0890 & 41.5 \\
$\beta_{1}$ & 5.474903380 & 0.8550 & 5920.9 \\
$\beta_{2}$ & -0.008164486 & 0.1317 & -22.5 \\
$\beta_{3}$ & -0.002097417 & 0.0660 & -8.2 \\
$\beta_{4}$ & -0.001591465 & 0.0842 & -5.5 \\
\hline
\end{tabular}

All five regression coefficients have large $t$ statistics indicating that with very high likelihood none of these coefficients is actually zero. From the last two coefficients (see Table 3), we can calculate the amplitude of the periodic fluctuation, and we get $A=4.4 \mu \mathrm{V}$. This is somewhat smaller than the $6.0 \mu \mathrm{V}$ we got when fitting the model to the four-watt data alone. This is not surprising since each power level has a different period. Thus, Eq. (7) is a simplification and a compromise - reality is more complicated. However, since most of the data came from the RH1979 (one-watt group) data, the simplified model should suffice here. From now on, we will ignore the last two coefficients; they are nuisance parameters. The values of the first three coefficients in Table 3 substituted into Eq. (2) pluvide the final calibration of the small calorimeter.

\section{Error Analysis}

Every determination of the thermal power of an unknown sample using calibration Eq. (2) is to some degree uncertain. This uncertainty stems from the following factors that are listed in a decreasing order of importance:

1. Random fluctuations in the (equilibrium) output voltage of the calorimeter

2. Long term periodic fluctuations in the output voltage

3. Uncertainty in the determination of the calibration coefficients $\beta_{0}, \beta_{1}$ and $\beta_{2}$

4. Modelling error, in the sense that Eq. (1) or Eq. (6) does not account for all nonrandom effects.

A standard measure of the uncertainty of an estimate is the mean squared error (MSE). If $w$ is the true value of thermal power, and $\hat{w}$ is the estimated power, then the mean squared error is defined as:

$$
M S E=E\left[(w-\hat{w})^{2}\right]=B I A S^{2}+\operatorname{Var}[(w-\hat{w})]
$$

Where the bias is defined as:

$$
B I A S=E[w-\hat{w}]
$$

Because many people think in terms of a fractional error (expressed in percent), we will use the following to relate MSE and fractional error: 


$$
\text { Error }=100 \times \frac{\sqrt{M S E}}{w}
$$

If we have the right statistical model that relates voltage to power, then the estimates of $\beta_{0}, \beta_{1}$ and $\beta_{2}$ will be unbiased, and if the measurement system does not drift, then the only bias in the estimate will be a mathematical bias because Eq. (2) is nonlinear in the random variables $v, \beta_{0}, \beta_{1}$ and $\beta_{2}$. However, since Eq. (2) is almost linear in $v$, the amount of mathematical bias should be nugligible. Nevertheless, we will check the magnitude of the mathematical bias to make sure.

\section{Error Variance}

Because we have chosen not to include the periodic signal in the calibration equation, we will treat it as if it were uncorrelated random noise, and inflate the variance assigned to the measured voltage, under the circumstances, this is a prudent procedure. The variance of the voltage measurement is given by:

$$
\sigma_{\nu}^{2}=\sigma_{\varepsilon}^{2}+\sigma_{\text {signal }}^{2}=\sigma_{\varepsilon}^{2}+\frac{A^{2}}{2}
$$

Where $A$ is the amplitude of the periodic signal, which we found to be proportional to the square of the thermal power. Thus, this component of the voltage variance will increase in proportion to the 4 th power of the thermal power.

If we expand Eq. (2) in a Taylor series, and apply the variance operator, then by keeping only linear terms, we get the following approximate expression for the variance of the calculated thermal power.

$$
\begin{aligned}
\operatorname{Var}(\hat{w})= & \left(\frac{\partial w}{\partial v}\right)^{2} \sigma_{\nu}^{2}+\left(\frac{\partial w}{\partial \beta_{0}}\right)^{2} \sigma_{\beta_{0}}^{2}+\left(\frac{\partial w}{\partial \beta_{1}}\right)^{2} \sigma_{\beta_{1}}^{2}+\left(\frac{\partial w}{\partial \beta_{2}}\right)^{2} \sigma_{\beta_{2}}^{2}+ \\
+ & 2\left\{\left(\frac{\partial w}{\partial \beta_{0}}\right)\left(\frac{\partial w}{\partial \beta_{1}}\right) \operatorname{cov}\left(\beta_{0}, \beta_{1}\right)+\left(\frac{\partial w}{\partial \beta_{0}}\right)\left(\frac{\partial w}{\partial \beta_{2}}\right) \operatorname{cov}\left(\beta_{0}, \beta_{2}\right)+\right. \\
& \left.+\left(\frac{\partial w}{\partial \beta_{1}}\right)\left(\frac{\partial w}{\partial \beta_{2}}\right) \operatorname{cov}\left(\beta_{1}, \beta_{2}\right)\right\}
\end{aligned}
$$

The partial derivatives are evaluated at the mean of the thermal. We can evaluate the partial derivatives in Eq. (9) by using the implicit function theorem to get expressions that involve only the thermal power level $w$, and the calibration coefficients $\beta_{0}, \beta_{1}$ and $\beta_{2}$; the results are as follows: 


$$
\begin{aligned}
& \frac{\partial w}{\partial v}=\frac{1}{\beta_{1}+2 \beta_{2} w} \\
& \frac{\partial w}{\partial \beta_{0}}=\frac{-1}{\beta_{1}+2 \beta_{2} w} \\
& \frac{\partial w}{\partial \beta_{1}}=\frac{-w}{\beta_{1}+2 \beta_{2} w} \\
& \frac{\partial w}{\partial \beta_{2}}=\frac{-w^{2}}{\beta_{1}+2 \beta_{2} w}
\end{aligned}
$$

\section{Calculation Mathematical Bias}

The calculation of the bias is somewhat more complicated because we need to apply the expectation operator to second order and cross-product terms in the Taylor Series expansion of Eq. (2). The cross-product terms lead to covariances. All covariance terms involving the voltage variable vanish because the fluctuations in the future voltage measurements must be statistically independent of the parameter estimates. The bias is given by Eq. (11) and the expressions for the second and mixed partial derivatives that follow.

$$
\begin{aligned}
& B I A S=\frac{1}{2}\left[\frac{\partial^{2} w}{\partial v^{2}} \sigma_{v}^{2}+\frac{\partial^{2} w}{\partial \beta_{0}^{2}} \sigma_{\beta_{0}}^{2}+\frac{\partial^{2} w}{\partial \beta_{1}^{2}} \sigma_{\beta_{1}}^{2}+\frac{\partial w}{\partial \beta_{2}^{2}} \sigma_{\beta_{2}}^{2}\right]+ \\
& +\frac{\partial^{2} w}{\partial \beta_{0} \partial \beta_{1}} \operatorname{cov}\left(\hat{\beta}_{0}, \hat{\beta}_{1}\right)+\frac{\partial^{2} w}{\partial \beta_{0} \partial \beta_{2}} \operatorname{cov}\left(\hat{\beta}_{0}, \hat{\beta}_{2}\right)+\frac{\partial^{2} w}{\partial \beta_{1} \partial \beta_{2}} \operatorname{cov}\left(\hat{\beta}_{1}, \hat{\beta}_{2}\right)
\end{aligned}
$$

The second and mixed partial derivatives are as follows: 


$$
\begin{aligned}
& \frac{\partial^{2} w}{\partial v^{2}}=\frac{-2 \beta_{2}}{\left(\beta_{1}+2 \beta_{2} w\right)^{3}} \\
& \frac{\partial^{2} w}{\partial \beta_{0}^{2}}=\frac{-2 \beta_{2}}{\left(\beta_{1}+2 \beta_{2} w\right)^{3}} \\
& \frac{\partial^{2} w}{\partial \beta_{1}^{2}}=\frac{2 w\left(\beta_{1}+\beta_{2} w\right)}{\left(\beta_{1}+2 \beta_{2} w\right)^{3}} \\
& \frac{\partial^{2} w}{\partial \beta_{2}^{2}}=\frac{4 \beta_{1} w^{3}+6 \beta_{2} w^{4}}{\left(\beta_{1}+2 \beta_{2} w\right)^{3}} \\
& \frac{\partial^{2} w}{\partial \beta_{0} \partial \beta_{1}}=\frac{\beta_{1}}{\left(\beta_{1}+2 \beta_{2} w\right)^{3}} \\
& \frac{\partial^{2} w}{\partial \beta_{0} \partial \beta_{2}}=\frac{2 w\left(\beta_{1}+\beta_{2} w\right)}{\left(\beta_{1}+2 \beta_{2} w\right)^{3}} \\
& \frac{\partial^{2} w}{\partial \beta_{1} \partial \beta_{2}}=\frac{3 \beta_{1} w^{2}+4 \beta_{2} w^{3}}{\left(\beta_{1}+2 \beta_{2} w\right)^{3}}
\end{aligned}
$$

\section{Error Calculations}

Equations (9), (10), and (11) together with the data from Table 3 and Table 4, suffice to calculate the fractional error in determining the power output of an unknown sample. We must also specify values for the amplitude of the periodic fluctuation and the parameters $\alpha_{0}$ and $\alpha_{1}$. The contribution of the mathematical bias to the total error was found to be less that $.01 \%$, and is therefore negligible. 
Table 4

Estimated of Covariances of First 3 Regression Coefficients from.Fa. (7)

\begin{tabular}{|c|cc|}
\hline Covariances $\times 10^{-6}$ & $\beta_{0}$ & $\beta_{1}$ \\
\hline$\beta_{0}$ & & \\
$\beta_{1}$ & -0.1549 & \\
$\beta_{2}$ & 0.0390 & -0.2810 \\
\hline
\end{tabular}

First, if we set $\alpha_{0}=0, \alpha_{1}=0$, and $A=0$, the resulting error is entirely due (absent modeling errors) to the uncertainty in the estimation of the coefficients of the calibration equation. We can see from the last column of Table 5 that this contribution is negligible below $10 \mathrm{~W}$.

Second, if we again set $A=0$, but put $\alpha_{0}=.0007, \alpha_{1}=.00302$ (from Fig. 5) then the contribution from the periodic signal is suppressed, but the inherent randam error of the measurement system is retained. This causes a substantial increase at the low powers (see Table 5 middle column).

Finally, changing $A$ to $0.006 \mathrm{mV} / \mathrm{W}^{2}$, we get the actual measurement error we can expect in practice. Note that for thermal powers above $1.0 \mathrm{~W}$, the contribution to the total error from the periodic signal is significant; for example at $4.0 \mathrm{~W}$, the periodic signal increases the error by a factor of five (see Table 5, second column). Since we lack data above $4.0 \mathrm{~W}$, the error calculations given in Table 5 at 10 and $20 \mathrm{~W}$ are extrapolations and may not be valid. In particular, we do not know that the amplitude of the periodic error signal will continue to grow as the square of the thermal power.

Table 5

Calibration Error by Power Level for Indicated Causes

\begin{tabular}{|c|c|c|c|}
\hline \multirow{2}{*}{ Power Level } & \multicolumn{3}{|c|}{ Error (percent) } \\
\hline & From All Effects & No Periodic Signal & Coefficient Uncertainty \\
\hline 0.25 & 0.10 & 0.11 & 0.02 \\
\hline 0.50 & 0.09 & 0.08 & 0.01 \\
\hline 1.00 & 0.10 & 0.07 & 0.01 \\
\hline 2.00 & 0.17 & 0.06 & 0.01 \\
\hline 4.00 & 0.31 & 0.06 & 0.02 \\
\hline 10.00 & 0.80 & 0.08 & 0.05 \\
\hline 20.00 & 1.65 & 0.14 & 0.13 \\
\hline
\end{tabular}

Note that the error tends to be minimal in the interval from 0.5 to $1.0 \mathrm{~W}$, the very place where we have most of our calibration data (see Fig. 6). 


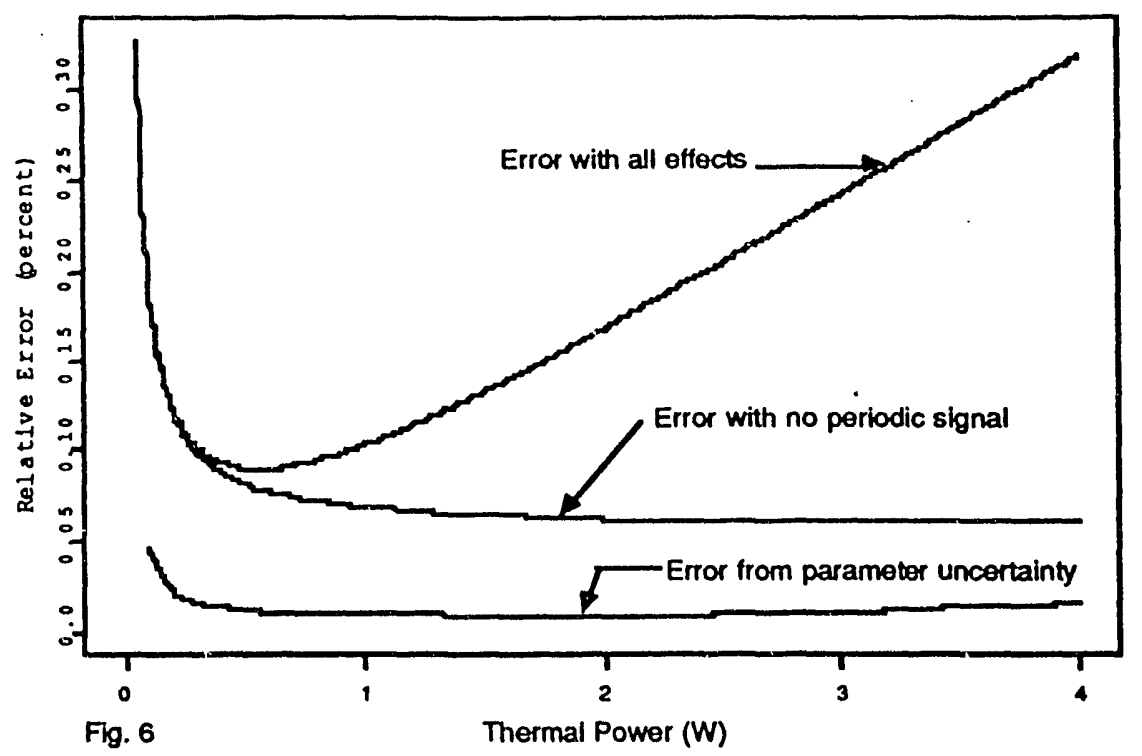

\section{Comparison of New Calibration with the Previous Calibration}

The prior calibration fit a cubic polynomial in the voltage variable to six data points; there are two problems with this procedure. First, the regression model uses power as the response variable and voltage as the predictor variable, and this will cause the estimates of the coefficients to be biased because the uncertainly lies mainly in the voltage. The procedure is also inefficient because the smoothing effect of using many measurements is lost. Since this data is well fit in any case by a straight line, the linear coefficient of the old and new calibrations should match. If we expand Eq. (2) in a Taylor series up to a cubic term, we can compare coefficients of the old and new calibrations. We can see from Table 7 that the constant and linear terms match, but the coefficients of the higher order terms do not, and it is the higher order terms that produce the high precision. Therefore, the new calibration should give an improvement in accuracy.

Table 7

Comparison of Old and New Calibration by Coefficients

\begin{tabular}{|c|c|c|}
\hline Term & Old Calibration & New Calibration \\
\hline Constant & -0.0030 & -0.0023 \\
\hline Linear & 0.1833 & 0.1826 \\
\hline Square $\times 10-6$ & -0.0605 & 0.4975 \\
\hline Cubic $\times 10-6$ & 3.4773 & 271.0134 \\
\hline
\end{tabular}

The greatest mismatch between the old and new calibrations is in the region from two to four watts (See Fig. 7), but calibrations will depart even more above four watts, but we do not know if either is valid. 


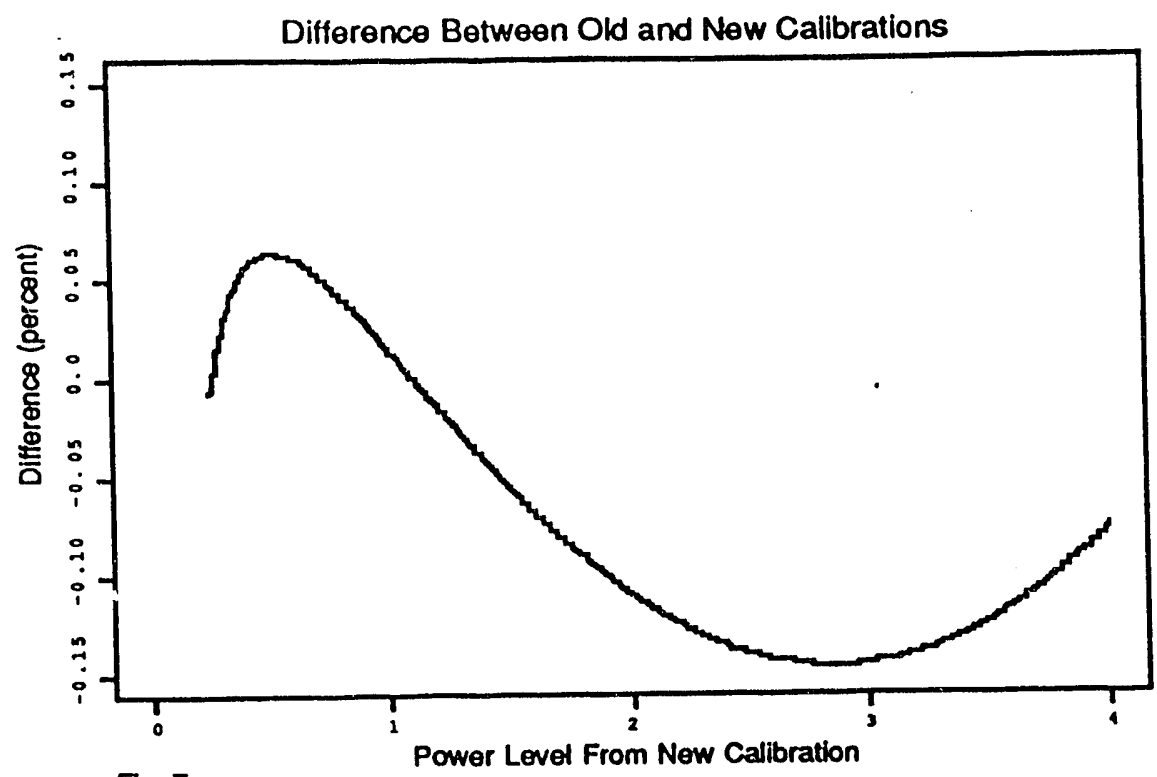

Fig. 7 


\section{References}

American National Standards Institute (1987). Plutonium-Bearing Solids Calibration Techniques for Calorimetric Assay. ANSI N15.22-1987.

Bloomfield, P. (1976). Fourier Analysis of Time Series: An Introduction. Wiley, New York.

Cleveland, W. S. (1979). Robust Locally Weighted Regression and Smoothing Scatterplots. Journal of the American Statistical Association. 74, 829-836.

Gunn, S. R. (1966). Some New Thermopile-Type Radiometric Calorimeters. Lawrence Livermore National Laboratory, Livermore, Ca, UCRL 50145.

Hoaglin, D. C., (1983). Letter Values: A Set of Selected Order Statistics. Understanding Robust and Exploratory Analysis. Wiley, New York.

Kendall, M. G., Stuart, A. S. (1977). The Advanced Theory of Statistics. Volume 1. Fourth Edition. Hafner, New York.

Steward, S. A., Tsugawa, R. T. (1975). Continued Studies of Calorimeter Performance at the Lawrence Livermore Laboratory. Lawrence Livermore National Laboratory, Livermore, Ca, UCRL 51964.

Tukey, J. W. (1977). Exploratory Data Analysis. Addison-Wesley, Reading MA. 

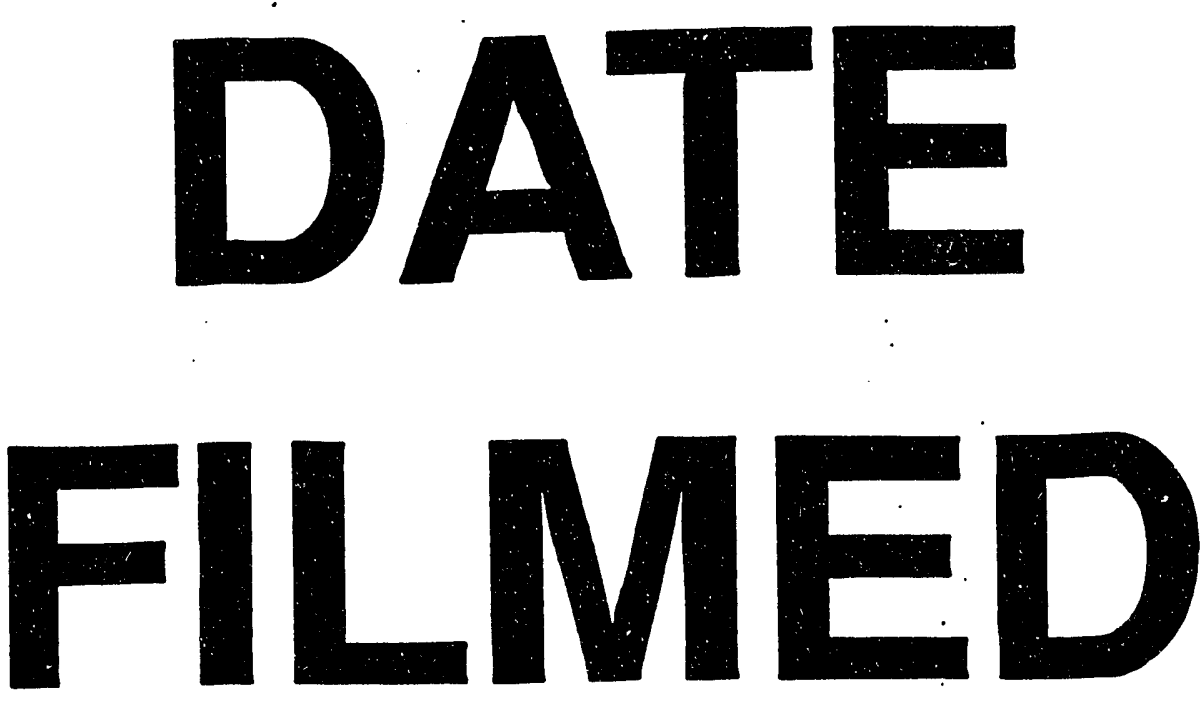

$11 / 9 / 93$
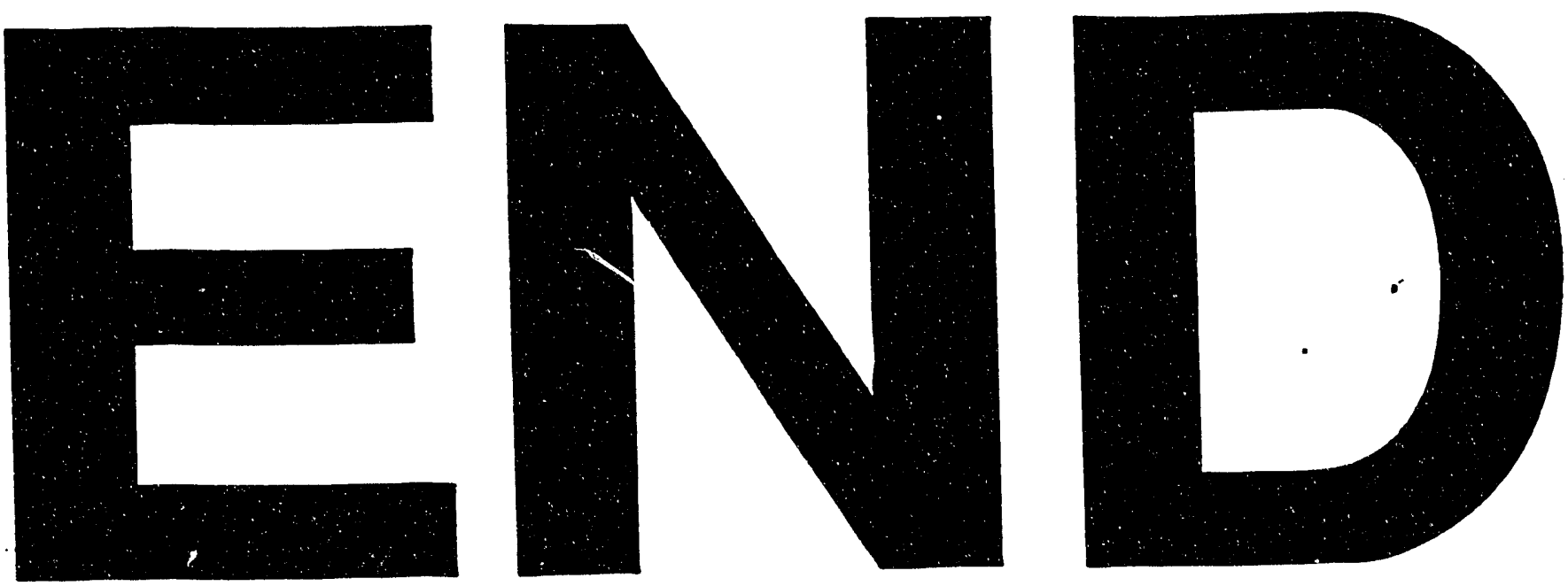
